\title{
Modeling capillary forces for large displacements
}

\author{
M. Mastrangeli, ${ }^{1, a)}$ Gari Arutinov, ${ }^{2,3}$ Edsger. C. P. Smits, ${ }^{2}$ and P. Lambert ${ }^{1}$ \\ ${ }^{1)}$ Department of Bio, Electro And Mechanical Systems (BEAMS), École Polytechnique de Bruxelles, \\ Université Libre de Bruxelles, Bruxelles (BE) \\ ${ }^{2)}$ Holst Center/TNO, High Tech Campus 31, 5656AE, Eindhoven (NL) \\ ${ }^{3)}$ Department of Mechanical Engineering, Eindhoven University of Technology, \\ Eindhoven (NL)
}

Originally applied to the accurate, passive positioning of submillimetric devices, recent works proved capillary self-alignment as effective also for larger components and relatively large initial offsets. In this paper we describe an analytic quasi-static model of $1 \mathrm{D}$ capillary restoring forces that generalizes existing geometrical models and extends the validity to large displacements from equilibrium. The piece-wise nature of the model accounts for contact line unpinning singularities ensuing from large perturbations of the liquid meniscus and dewetting of the bounding surfaces. The superior accuracy of the generalized model across the extended displacement range, and particularly beyond the elastic regime as compared to purely elastic models, is supported by finite element simulations and recent experimental evidence. Limits of the model are discussed in relation to the aspect ratio of the meniscus, contact angle hysteresis, tilting and self-alignment dynamics.

\section{INTRODUCTION}

Liquid bridges connecting adjacent surfaces ${ }^{1,2}$ find extensive use in assembly ${ }^{3}$ and precision engineering ${ }^{4}$. A common example is represented by a liquid droplet bridging a flat (e.g. surface-mount ${ }^{5-7}$ ) component onto a stationary bottom surface. The position of the dropletand hence that of the floating component-can be constrained by patterning a receptor site onto the substrate ${ }^{8}$. A single state of globally minimal energy exists for this capillary system ${ }^{9}$, provided that the surface of the component matches in shape and size that of the bottom site and that the volume of the droplet is sufficiently small to avoid tilting ${ }^{10,11}$. In this equilibrium state the component stands parallel to the substrate and its edges are aligned to those of the site ${ }^{9}$. Upon formation of the vertical liquid bridge, capillary forces ${ }^{9,12}$ and torques ${ }^{9,13,14}$ act on the component. Capillary self-alignment refers to the motion of the component towards the absorbing state across an initial lateral offset. The same capillary forces tend to restore the system into the equilibrium state against perturbations and displacements ${ }^{8}$. Selfrecovery of rest position and intrinsic mechanical compliance sparked interest in droplet-based capillary systems for relevant technological applications, such as conformal joints $^{15-17}$, compliant handling ${ }^{4}$, precise registration of components $^{18,19}$ and self-assembly ${ }^{8}$. Thereby the focus has mainly been on sub-millimetric components actuated by droplets of similar size, and correspondingly on displacements from equilibrium of relatively small magnitude. Elastic models accurately capture the response of the capillary system to such small lateral displacements ${ }^{9,12,20-22}$. In this type of models the restoring capillary forces arise exclusively from the shear deformation of liquid interfaces pinned to bounding solid surfaces. Recently however capillary self-alignment was successfully

\footnotetext{
${ }^{a)}$ Corresponding author: massimo.mastrangeli@ulb.ac.be
}

demonstrated also for centimeter-sized components ${ }^{7,23}$ across relatively large lateral offsets ${ }^{24,25}$. Accounting for such evidence prompts an expansion of the reach of purely elastic models through wetting arguments.

In this paper we present a generalized quasi-static model of lateral capillary restoring forces valid for a significantly extended range of relative component displacements as compared to elastic models. By accounting for both liquid meniscus' partial wetting of solid surfaces (earlier attempted $^{26,27}$ ) and limited angle hysteresis over edges, the model portraits more accurately the behavior of finitely-deformable solid/liquid interfaces. For displacements larger than the elastic limit, we show that this behavior is distinctively characterized by the unpinning of triple contact lines. These wetting discontinuities are integral to the presented model, and supported by recent experimental evidence ${ }^{24,25}$. We claim for the first time that the discontinuities divide the capillary response of the meniscus into three sequential regimes and pose the physical justification of the piece-wise nature of the model. The proposed model coincides with the purely elastic one for small displacements, and it describes the system with signicantly better accuracy otherwise. It thus bridges the gap with abstract geometrical models ${ }^{26}$ which tend to hold in the domain of large displacements.

An intuitive formulation of the model and its finite element simulation are presented respectively in Sect. II A and II B. Comparative results are illustrated in Sect. III, followed by a discussion of experimental support and limitations of the model (Sect. IV), and by conclusions (Sect. V). Full derivation of the model is described in the Appendix.

\section{MODEL FORMULATION}

\section{A. Analytical model}

We consider the system geometry sketched in figure 1 . We refer to the solid bounding objects as pads, and to 

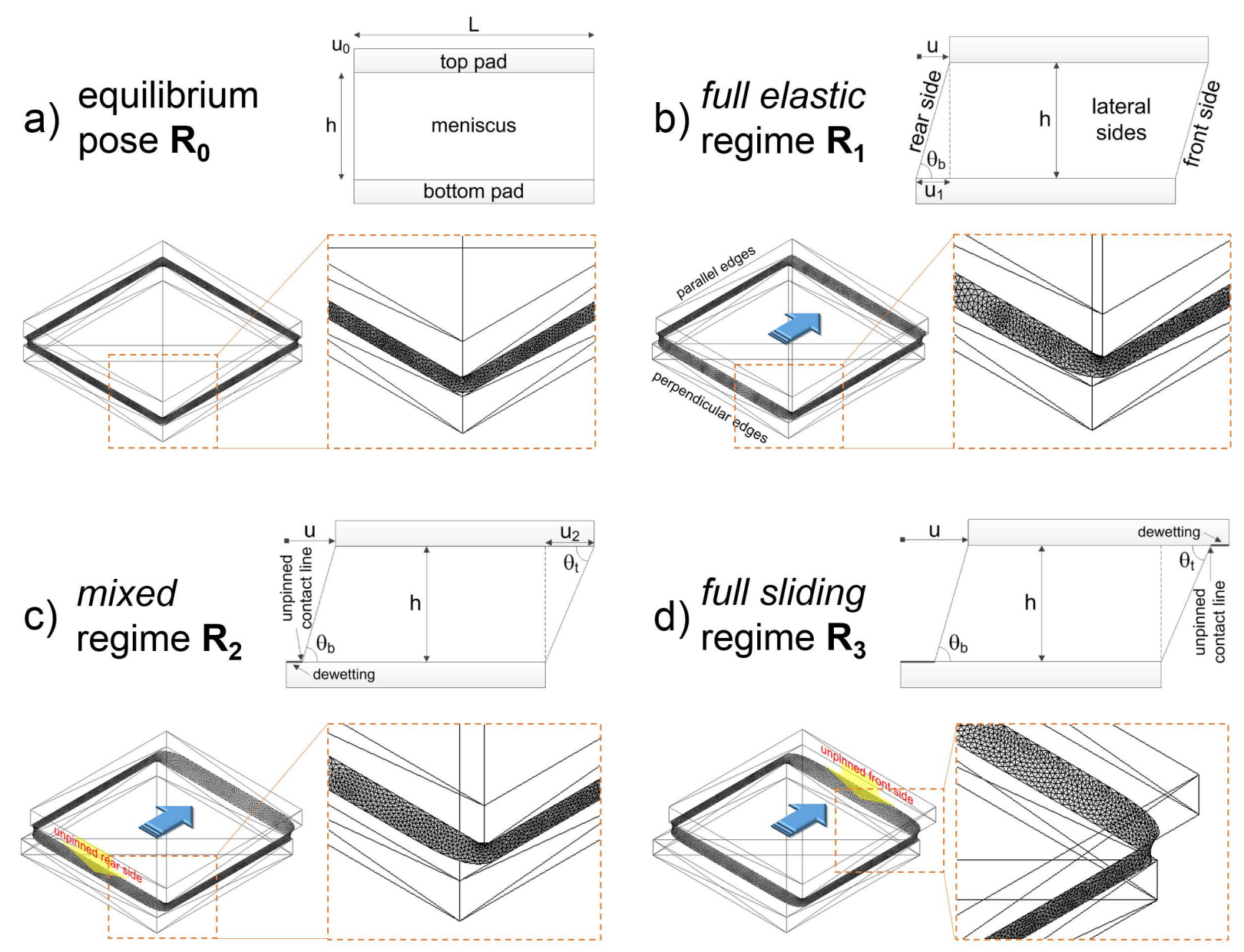

FIG. 1. Geometrical sketches (upper rows, not to scale) and SE snapshots with inset views (lower rows, $L=1 \mathrm{~mm}, h=50 \mu \mathrm{m}$ ) of sequential regimes $R_{j}$ with $0<\theta_{t}<\theta_{b}<\pi / 2$. a) Global equilibrium, b) full elastic regime, c) mixed regime, d) full sliding regime. During capillary self-alignment the regimes are traversed in the reverse order with respect to model derivation.

the liquid bridge interchangeably as meniscus. Top $(t)$ and bottom $(b)$ pads have the same square shape of sidelength $L$. The meniscus has constant volume $V$, density $\rho$, surface tension $\gamma$ and height or gap $h$. Starting from the equilibrium position $u_{0}=0$ (figure 1a), a quasi-static horizontal displacement $u>u_{0}$ of the top relative to the bottom pad is imposed along a main orthogonal direction (figure 1b). Null relative tilt of the top pad is assumed for all $u$ values considered. Lateral, front and rear sides of the meniscus, and parallel and perpendicular edges of the pads, are defined by their respectively parallel or perpendicular orientation with the direction of deformation (refer to figure 1b). The shearing perturbation $u$ induce an asymmetrical deformation of the meniscus. The model describes the capillary response of the system to increasing deformations.

The following simplifying model assumptions are used:

1. quasi-static equilibrium, i.e. the system is originally in global equilibrium and arbitrarily close to local equilibrium in every perturbed configuration. Inertial and viscous effects are therefore neglected by assuming $W e \ll 1$ and $C a \ll 1$, respectively;

2. constant gap $h(u)=h_{0}=h$, and smaller than the capillary length $L_{c}=\sqrt{\gamma / \rho g}$ (i.e. $\left.B o \ll 1\right)$ to neglect gravitational effects;

3. ideally straight surfaces of meniscus sides, including negligible weight of the top pad;

4. ideal smoothness and chemical homogeneity of solid surfaces, i.e. null contact angle hysteresis except along edges, and smooth unpinning of triple contact lines upon dewetting;

5. no liquid overflow beyond pad edges.

Given the previous hypotheses, which define the quasistatic model framework, contact angles $\theta_{*}$ can be defined everywhere on each solid surface (except over discontinuities such as edges) by the corresponding Young-Dupré equation $^{28}$ :

$$
\gamma_{\mathrm{sv}}^{*}-\gamma_{\mathrm{sl}}^{*}=\gamma \cos \theta_{*}
$$


where the pedexes $s, l, v$ refer to solid, liquid and vapor phases, respectively, and $*$ stands for $t$ and $b$. Top and bottom pads are partially wetting. They have differing interfacial energies $\gamma_{\mathrm{sl}}^{*}$ and $\gamma_{\mathrm{sv}}^{*}$ for generality, and all its sides have the same surface energies. Given the contact angles $\theta_{b} \neq \theta_{t}$ (with $0<\theta_{*}<\pi / 2$ ), we define $\theta_{\min }=$ $\min \left(\theta_{t}, \theta_{b}\right)$ and $\theta_{\max }=\max \left(\theta_{t}, \theta_{b}\right)$.

Upon relative displacement $u>0$ of the pads the meniscus deforms to accomodate the shear stress. The capillary reaction against the perturbation is mainly exerted through the front and rear sides of the meniscus. For increasing values of $u$, the reaction assumes one of two sequential types depending on the degree of meniscus deformation. For each of the perpendicular sides of the meniscus, the transition between the two types of reaction is signaled by the unpinning of one contact line. At pad level, the contact line that undergoes unpinning is specifically the one on the perpendicular edge subtending the smallest edge angle. The opposite edge bears no unpinning since overflow is geometrically avoided according to hypothesis 5 (and as detailed below). The elastic reaction takes place before this discontinuity. In the elastic response the sides stretch their surfaces and incline forward due to edge angle hysteresis. Over the edges of the pads the contact lines work like hinges maintaining their position fixed. Unpinning is prompted by reaching the receding value of the contact angle, here coinciding by assumption with $\theta_{*}$. Beyond this point, the shear deformation of the meniscus is accomodated by its unpinned side(s) by sliding the unpinned contact line across the surface of the pad. In this sliding response the surface area and inclination of the unpinned side(s) remain constant.

The complete response of the meniscus as function of the relative displacement $u$ of the pads is thus divided into three sequential regimes $R_{j}$. In the first regime $R_{1}$ (full elastic, figure $1 \mathrm{~b}$ ), both perpendicular sides of the meniscus enact elastic response and have the same inclination; in the second regime $R_{2}$ (mixed, figure 1c), the perpendicular sides enact either responses; in the third and last regime $R_{3}$ (full sliding, figures $1 \mathrm{~d}$ ) both perpendicular sides are sliding. The separation between regimes is intrinsically set by the unpinning discontinuities. The discontinuities tend to happen for the same $u$ instead of sequentially-i.e. the second regime disappears-in the limit of equal surface energies of the pads. For each of the perpendicular sides, the elastic and sliding force components have respectively the form (see Appendix) $F_{e l}=\gamma L \frac{u}{\sqrt{h^{2}+u^{2}}}$ and $F_{s l}=\gamma L \cos \theta_{*}$. Hence the capillary restoring force $F(u)$ is described by the following piece-wise equation:

$$
F(u)= \begin{cases}-2 \gamma L \frac{u}{\sqrt{h^{2}+u^{2}}} & 0<u<u_{1} \\ -\gamma L\left(\cos \theta_{\max }+\frac{u}{\sqrt{h^{2}+u^{2}}}\right) & u_{1}<u<u_{2} \\ -\gamma L\left(\cos \theta_{\max }+\cos \theta_{\min }\right) & u_{2}<u<u_{\max }\end{cases}
$$

where $u_{\max }$ is defined in Appendix and relates $u$ to $L$

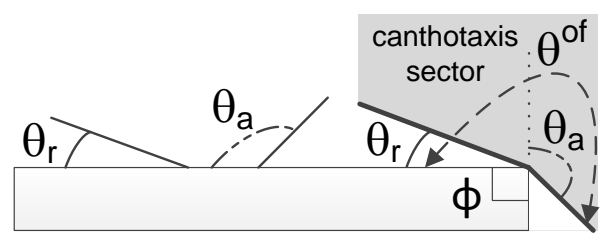

(a) Edge confinement by topographical step.

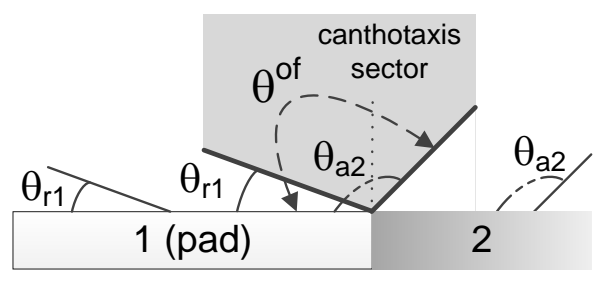

(b) Edge confinement by wetting contrast.

FIG. 2. Meniscus confinements by edge angle hysteresis for $\theta_{r} \neq \theta_{a}$. For case (a) developed in the text the quasi-static model assumes $\theta_{r}=\theta_{a}=\theta_{*}$ and the canthotaxis spans an angular sector of $\pi-\phi$ (see eq. 4). Case (b) is considered in the Appendix.

rather than to $h$. The domain boundary values:

$$
u_{1}=h \cot \theta_{\max } \text { and } u_{2}=h \cot \theta_{\min }
$$

can be obtained by imposing force continuity between adjacent regimes (smooth transitions, hypothesis 4). They satisfy the geometric interpretation shown in figures $1 \mathrm{~b}$ and $1 \mathrm{c}$, respectively.

Overflow (of) avoidance (hypothesis 5) sets additional contraints on $u$ and $\theta_{*}$. The liquid meniscus is constrained within the edges of top and bottom pads. Along the pad edges the contact line is pinned and the angles formed by the meniscus with the surfaces of the pads (i.e. edge angles) can assume a multiplicity of values. Edge angle hysteresis is measured by the range of angles coexisting over the same contact line position (i.e. the canthotaxis sector ${ }^{28}$, figure 2). While the lower limit of the edge angle coincides with the receding contact angle $\theta_{r}^{*}$, the higher limit $\theta^{o f}$ is determined by case-specific boundary conditions. Exceeding the higher limit of edge angles prompts overflow of the liquid bridge beyond the edges of the pads. This evenience represents a major failure mode for capillary self-alignment ${ }^{8}$ - and coincides with the ultimate limit of validity of the model. Meniscus confinement can be enforced chemically or topographically. In the former case, surface chemistry is tailored to make the pads more wettable than the surrounding $\operatorname{areas}^{23}$, and $\theta^{\text {of }}$ is 
set by the advancing contact angle of the surrounding area (figure 2(b)). In latter case (figure 2(a)) the canthotaxis sector is extended by edge confinement ${ }^{29,30}$, and $\theta^{\text {of }}$ is imposed by the Gibbs' criterion ${ }^{12}$ :

$$
\theta^{o f}=\theta_{*}+\pi-\phi
$$

$\phi$ being the slope angle of the pad's edge. Accordingly, overflow takes place when the edge angle reaches the value of the advancing contact angle with respect to the surface of the pad's sloped side. In the following we consider and model the case of topographical confinement with vertical pad sides (i.e. $\phi=\pi / 2$, figure $2 \mathrm{a}$ ). The analytical model for the chemical confinement case can be similarly derived, and is described in the Appendix.

Consistency with the Gibbs' criterion (eq. 4) to avoid meniscus overflow imposes coupled constraints on the values of $\theta_{*}$ with respect to eq. 3 . Specifically, transitions between $R_{1}$ and $R_{2}$ and between $R_{2}$ and $R_{3}$ can take place without overflow for corresponding $u_{i}$ only if:

$$
\begin{cases}\theta_{\min } \geq \pi / 2-\theta_{\max } & R_{1} \text { to } R_{2} \\ \theta_{\max } \geq \pi / 2-\theta_{\min } & R_{2} \text { to } R_{3}\end{cases}
$$

These model boundary conditions prescribe a mutual relation between the surface energies of the pads for physical consistency. Hence overflow avoidance implies that the full sequence of reaction regimes be conditional to the coupled choice of $\theta_{*}$. The case of perfect pad wettability, normally assumed in elastic models, is trivially excluded in presence of edge confinement, and only possible for chemical confinement. Liquid overflow due to enhanced wettability of pads with topographical edge confinement was recently evidenced in self-alignment experiments ${ }^{25}$.

\section{B. Finite element model}

The capillary system was simulated by a quasi-static finite element numerical model in Surface Evolver ${ }^{31}$ (SE) (figure 1). Water ${ }^{7,19,23}(\gamma=72 \mathrm{mN} / \mathrm{m}, \rho=$ $1000 \mathrm{~kg} / \mathrm{m}^{3}, L_{c}=2.7 \mathrm{~mm}$ ) was chosen for the liquid bridge constrained within square pads of $L=1 \mathrm{~mm}$ $\left(B o=\rho g L^{2} / \gamma=0.14\right)$, thickness of $125 \mu \mathrm{m}$ and density of $1380 \mathrm{~kg} / \mathrm{m}^{3}$. Domain constraints were used to confine the triple contact lines of the meniscus strictly in the planes and within the perimeters of the pads. The weight of the top pad $(173 \mu \mathrm{g})$ had negligible impact on the model. The simulations proceeded by the displacement of the top pad within the range $u_{0}<u<u_{\lim }$ in steps of $1 \mu \mathrm{m} . u_{\max }<u_{\lim }<L$ was chosen to check the limits of validity of the model within a physically realistic range of $u$. Values of $\left.h\right|_{u_{0}}=25,50$ and $100 \mu \mathrm{m}$ were simulated using corresponding values of liquid volume $V=25,50$ and $100 \mathrm{~nL}$. They correspond to $h / L$ aspect ratios (AR) of $1 / 40,1 / 20$ and $1 / 10$, respectively, spanning the AR range used in applications. Several combinations of $\theta_{b}$ and $\theta_{t}$ values were simulated for each $\mathrm{AR}$, with $\theta_{*} \subset\left\{0^{\circ}, 10^{\circ}, 35^{\circ}, 50^{\circ}, 65^{\circ}, 80^{\circ}\right\}$. The case of perfect wetting $\left(\theta_{*}=0^{\circ}\right)$ was considered, in spite of its inconsistency with edge confinement (eq. 5), as reference for the purely elastic scenario. $u_{\max }$ was defined for each $\theta_{*}$ pair and $h$ values according to eq. 12 (see Appendix). Mesh refinement and geometry evolution made repeated use of the built-in Hessian of the energy function. Energy convergence was assumed to be attained at its fifth significant digit for all simulated configurations. The SE model complied with all assumptions stated in Sect. II A, except for the straight surface of the sides of the meniscus (see figure 1) and the constancy of $h(u)$. After every update of $u, h(u)$ was updated through a local-search routine based on Newton's method to keep the system in local equilibrium ${ }^{15}$. The non-constancy of $h(u)$ was implemented in SE for physical consistency with 1) the time scale separation due to the dominance of vertical over lateral capillary forces for this system ${ }^{26}, 2$ ) the tendency of the liquid bridge to assume the geometry locally closest to a section of a sphere ${ }^{15,28}$, and 3) conservation of the meniscus volume, which upon contact line unpinning(s) induces an increase of $h$ to partially compensate the decrease of wet pad surface(s). After setting $h(u)$, the lateral capillary force $F(u)$ was computed by the method of virtual works ${ }^{32}$ implemented using central finite differences.

\section{RESULTS}

SE simulations evidenced the continuity and monotonicity of $E(u)$ and $h(u)$, and their sensitivity to the energy of the solid surfaces as lumped in the $\theta_{*}$ pairs (see figures 3(a) and 3(b), respectively, for $h_{0}=25 \mu \mathrm{m}$ ). Higher surface energies (i.e. lower $\theta_{*}$ ) yield higher total system energies and energy gradients (i.e. forces, shown below) as well as smaller variations in $h(u)$. As for the latter, values of $\theta_{*}>0$ induce an increase of $h(u)$, whereas perfect wetting $\left(\theta_{*}=0^{\circ}\right)$ causes a decrease.

An instance of the capillary forces predicted by the analytical and SE models is shown in figure 4 . For this case $\left(h=25 \mu \mathrm{m}, \theta_{b}=65^{\circ}\right.$ and $\left.\theta_{t}=50^{\circ}\right)$ all regimes are encountered. The sequential unpinning discontinuities are seen to coincide with the sharp beginning of linear decrease of the wet areas $A_{*}$ of the pads. The accuracy of the match between proposed and SE model across all range of $u$ can be appreciated - particularly with respect to the purely elastic model represented by $F_{1}(u)$. The small deviations are attributed to the non-constancy of $h(u)$ and to the hysteresis of edge angles simulated in SE. As the gap increases with $u$ the lateral sides of the meniscus may deform and curve, contributing elastically to the total energy and the ensuing capillary force in ways not accounted for analytically. The edge angle hysteresis in SE is larger than what considered in the analytical counterpart. Such hysteresis retards the unpinning of the contact lines. Consequently the $u_{i}$ predicted by the analytical model tend to be smaller than the simulated ones - a relatively small error over $\theta_{*}$ may produce 


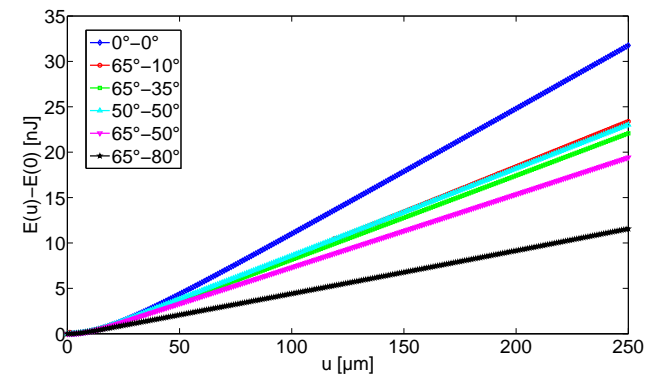

(a) Total energy $E(u)-E(0)$ versus $u$

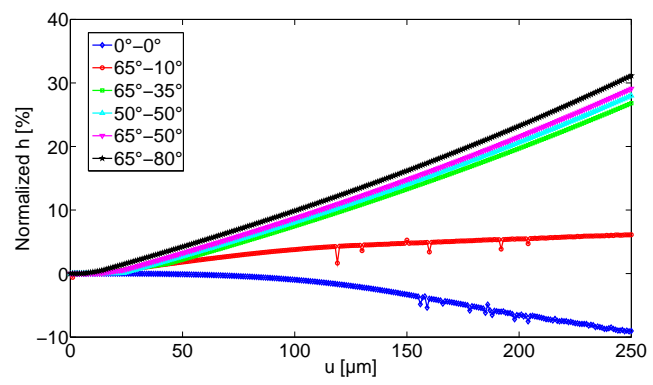

(b) normalized gap $\left(h-h_{0}\right) / h_{0}$ versus $u$

FIG. 3. SE simulations for $h_{0}=25 \mu \mathrm{m}(\mathrm{AR}=1 / 40)$ parameterized by $\theta_{b}-\theta_{t}$ pairs.

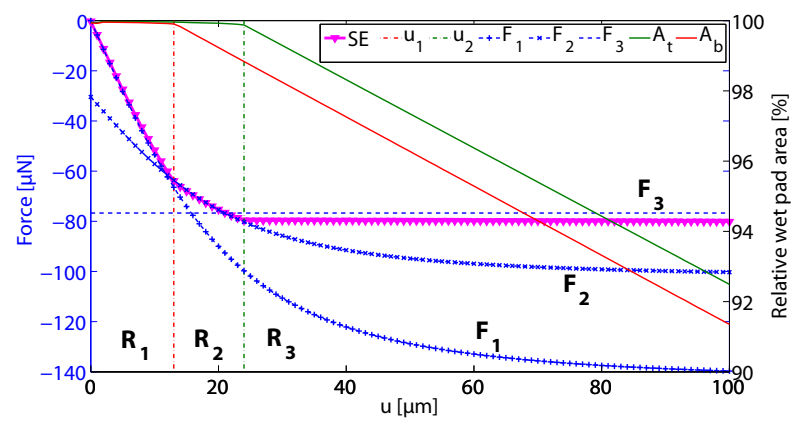

FIG. 4. SE and analytical predictions of $F(u)$ versus $u$ for $h_{0}=25 \mu \mathrm{m}(\mathrm{AR}=1 / 40)$ with $\theta_{b}=65^{\circ}$ and $\theta_{t}=50^{\circ}$. Vertical lines correspond to $u_{i}$ and signal contact line unpinning from pad edges by the decrease in wet pad area $\left(A_{*}\right)$. Analytic forces $F_{j}$, holding in respective domains $R_{j}$, are shown across the entire domain of $u$ for comparison. Relative error for $F\left(u=u_{\max }\right)$ is $4.3 \%$.

large effects given that $\frac{\partial u_{i}(h, \theta)}{\partial \theta}=-\frac{h}{\sin ^{2}(\theta)}$. The force predicted, particularly for large displacements, is hence cumulatively affected by both unaccounted effects. For instance, for the case of figure 4 the analytical values of $u_{1}=11.7$ and $u_{2}=21 \mu \mathrm{m}$ compares with $u_{1}=13$ and $u_{2}=24 \mu \mathrm{m}$ obtained from SE, leading to a relative error of about $4 \%$ over the value of $F_{3}\left(u_{\max }\right)$. Figure 5 further exemplifies the verification of the model for other cases with $h=25 \mu \mathrm{m}$ and significant combinations of $\theta_{*}$ values. The error over $F_{3}(u)$ remains small and bounded for larger $\theta_{*}$, while that of the purely elastic model increases.

\section{DISCUSSION}

As shown by the relative errors over $F\left(u_{\max }\left(\theta_{*}, h\right)\right)$ summarized in Table I, for larger AR values the discrepancy between analytical and SE models increases, and several effects undermine the validity of the former. As mentioned in the previous section, SE simulations show that for receding contact lines the edge angle hysteresis tend to slightly deviate from the analytical description. This may be attributed to the finite length of the pads in the direction perpendicular to $u$, since the ensuing curvature of the contact line is not considered in the derivation of Gibbs' criterion ${ }^{12}$. Moreover, the hypothesis of straight meniscus sides holds fairly well in SE except expectedly at the pads' corners (figures $1 \mathrm{~b}-1 \mathrm{c}$ ). This is permitted by and consistent with the updating of $h(u)$ by time scale separation (see Sect. II B) and, importantly, the unpinning of the contact lines upon reaching the limits of the elastic responses. Contact line unpinning allows preserving the inclination of the unpinned meniscus sides independently of further increments of $u$-in contrast to purely elastic and perfect wetting cases. Yet the curvature and deformation of the sides of the meniscus becomes evidently less negligible as $u$ and AR increase. As a result, contact line unpinning becomes less sharp and resembles unzipping, as it takes place not at once but rather gradually, starting from the extremities of the edges toward the center. This fuzzyfies the boundaries between the regimes, since the $u_{i}$ can not be univocally identified. Therefore the model tends to loose accuracy for increasing values of AR besides of $u$.

It can additionally be argued by the Hauksbee principle $^{28}$ that for relative displacements of the order of $u_{\max }$ the condition of strictly horizontal translation of the top pad (see Section II A) may break down, as tilting of the same may intervene to accomodate the relevant perturbation of the meniscus. Together with meniscus overflow, tilting is reportedly a significant failure mode in capillary self-alignment ${ }^{8}$. In presence of tilting the self-aligning process is compromised because the capillary forces are opposed by friction between solid surfaces ${ }^{11,18,23}$. Moreover, the tilt mode provides limited restoring torque ${ }^{9}$, and the system may effectively escape from the state of local energy minimum only through external agitation $^{11,22}$. The incidence of tilting can be lessened and even avoided by optimizing the volume ${ }^{10,23}$ and wetting ${ }^{24}$ of the liquid meniscus - as confirmed by the experimental evidence provided below and, incidentally, by the disappearance of pad tilt during evaporation of the meniscus ${ }^{19}$.

The models, consistently with earlier instances, follow the progressive displacement of the capillary system from its state of global equilibrium opposed by restoring forces. Hereby the work of displacement is used to stretch the meniscus and eventually dewet solid surfaces. Through this approach, our extended model allows to predict the parabolic regime we earlier reported in experimental high-resolution tracking of capillary selfalignment dynamics ${ }^{24}$. The parabolic regime ensues from 


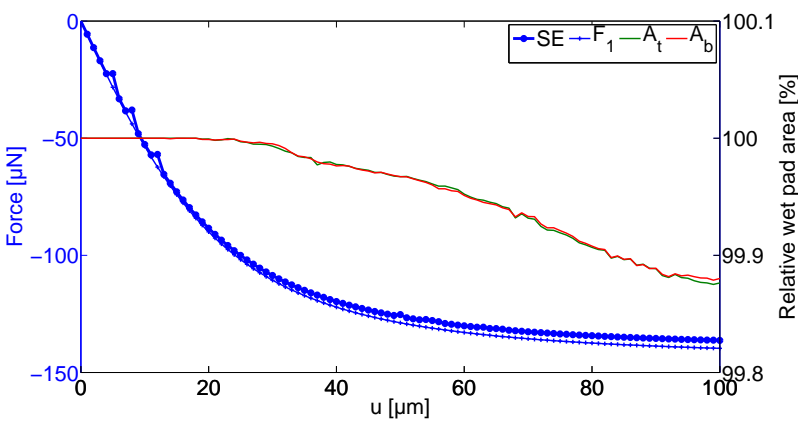

(a) $\theta_{b}=\theta_{t}=0^{\circ}$

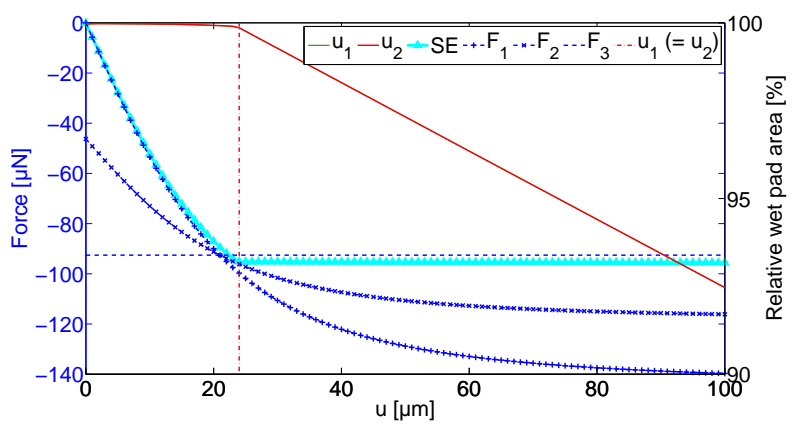

(c) $\theta_{b}=\theta_{t}=50^{\circ}$

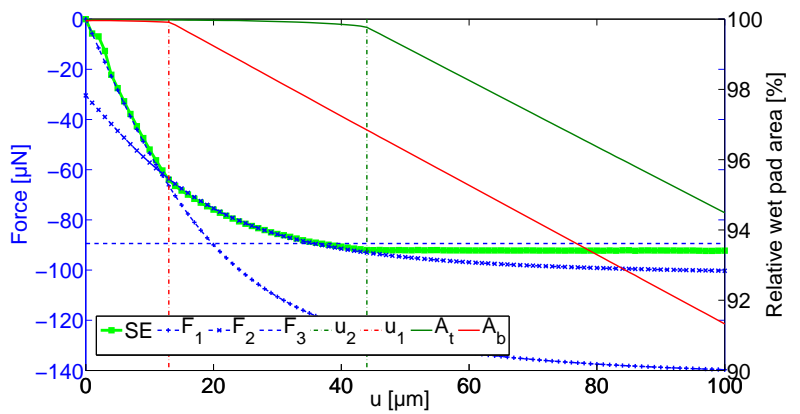

(b) $\theta_{b}=65^{\circ}, \theta_{t}=35^{\circ}$

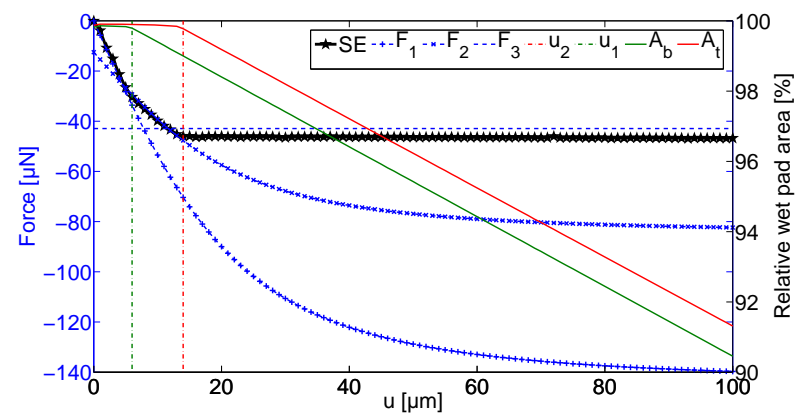

(d) $\theta_{b}=65^{\circ}, \theta_{t}=80^{\circ}$

FIG. 5. Capillary forces predicted for $h=25 \mu \mathrm{m}(\mathrm{AR}=1 / 40)$ and various combinations of $\theta_{*}$. (a) Perfect wetting (no contact line unpinning), (b) the three sequential regimes, first unpinning on bottom pad, (c) absence of second regime for $\theta_{b}=\theta_{t}$ $\left(u_{1}=u_{2}=u_{d}, F_{1}\left(u_{d}\right)=F_{2}\left(u_{d}\right)=F_{3}\left(u_{d}\right)\right)$, (d) the three sequential regimes, first unpinning on top pad.

TABLE I. Relative errors for $F\left(u_{\max }\left(\theta_{*}, h\right)\right)^{\text {a }}$

\begin{tabular}{|c|c|c|c|}
\hline$\theta_{b}-\theta_{t}$ & $h_{0}=25 \mu \mathrm{m}$ & $h_{0}=50 \mu \mathrm{m}$ & $h_{0}=100 \mu \mathrm{m}$ \\
\hline \hline $0^{\circ}-0^{\circ}$ & $2.7 \%$ & $4.2 \%$ & $7.4 \%$ \\
$65^{\circ}-10^{\circ}$ & $2.8 \%$ & $5.5 \%$ & $16 \%$ \\
$65^{\circ}-35^{\circ}$ & $3.1 \%$ & $4.3 \%$ & $7 \%$ \\
$50^{\circ}-50^{\circ}$ & $3.2 \%$ & $5.4 \%$ & $3.7 \%$ \\
$65^{\circ}-50^{\circ}$ & $4.3 \%$ & $7.3 \%$ & $7.4 \%$ \\
$65^{\circ}-80^{\circ}$ & $8.6 \%$ & $14.7 \%$ & $17.1 \%$ \\
\hline
\end{tabular}

a See eq. 12 in the Appendix for the numerical values of $u_{\max }$.

a constant acceleration imparted by the relaxing liquid bridge to the top pad. The constant acceleration is consistent with the constant restoring capillary force predicted by eq. 2 for large relative displacements.

However, in its present form the proposed model can not frame the actual experimental dynamics of capillary self-alignment ${ }^{24}$. This can be illustrated through sequential snapshots from a high-speed recording of a capillary self-alignment experiment, shown in figure 6 . In this realization a transparent top pad (polyethylene naphthalate, water contact angle $\theta_{t}=65^{\circ}$ ) was controllably dropped onto a thin water layer $(h=125 \mu \mathrm{m})$ pre-coated over the entire bottom receptor site (silicon dioxide surface, $\theta_{b}=10^{\circ}$ ) except for its corners ${ }^{8}$ (figure 6(a)). Chemical edge confinement was used as the bottom pad was surrounded by non-wetting areas with water contact angle $\theta_{o f}=120^{\circ 23}$. Edge confinement is exemplified elsewhere ${ }^{25}$. In spite of the optimized precoating $^{23}$, conformal coverage of both pads by the liquid bridge is achieved along the self-alignment process only after a sequence of dynamic regimes. In the initial transient wetting regime ${ }^{24}$ the meniscus deforms and spreads until its contact lines pin on the edges of the pads $^{33}$ (figure 6(b)). Only then can the capillary forces exerted by the meniscus be applied to the pads, causing their relative translation. Henceforth the self-aligning motion starts and proceeds along a predictable spatiotemporal trajectory across the aforementioned parabolic regime (figure 6(c)) and subsequent underdamped harmonic oscillations ${ }^{24}$ (figure 6(e)). In case of partial wetting, it is only after crossing the receptor site for the first time that the surfaces of both pads are fully wet by the liquid bridge (figure 6(d)). After transient wetting, the front side of the meniscus was pinned throughout the process on both pads. Hence the mixed $R_{2}$ regime predicted by the model extended from the inception of the translational motion of the top pad through the constant acceleration regime (figure 6(c)) till first crossing of the target position. The rear side of the meniscus remained unpinned, as expected from the model. Fully elastic regime $R_{1}$ appeared during all subsequent overshoots conducing the oscillating top pad to the final equilibrium position in accurate alignment with the bottom site (figure 6(f)). 
Notably, in our consistently repeatable experiments ${ }^{25}$ no tilt of the top pad was recorded even for very large initial offsets $^{25}(1.5 \mathrm{~mm}$ in the illustrated instance, i.e. about $30 \%$ of $L)$-i.e. also for offsets larger than the limiting value of $u_{\max }$ correspondingly estimated in the model. This evidence further suggests that the incidence of tilt is also significantly dependent on the contingent dynamics through which the rest position is approached.

Importantly, during the actual dynamics of selfalignment the reaction regimes $R_{j}$ described by the proposed model are traversed in a different, generally reverse temporal sequence, so that $R_{0}$ represents the absorbing state of the dynamics instead of the initial state as assumed in the modeling. A comprehensive dynamic model of the process would therefore need to consider advancing rather than receding contact angles (dependent on line velocity ${ }^{34}$ ) in the sliding regimes - together with inertia, fluid flow and viscosity ${ }^{20}$ and possibly energy dissipation at the moving contact lines ${ }^{34}$. The proposed model provides an extended quasi-static scenario toward the development of such as yet elusive ${ }^{20,24}$ description.

\section{CONCLUSIONS}

We introduced an analytic description of restoring capillary forces accounting for an extended range of lateral perturbations of a confined liquid bridge. The proposed model provides a simple and accurate description of the capillary system by integrating the effect of partial wetting of the bounding solid surfaces by the liquid bridge. Thanks to this, important physical and geometrical parameters of the system-such as surface energies, pad dimensions and type of edge confinement against liquid overflow - are additionally accounted for, which further distinguishes the present from earlier models. The analytic description is supported by physically consistent finite element simulations. The numerical simulations also highlight the physical limits of validity of the model, coherently with its stated assumptions and boundary conditions. Predictions concerning the effect of surface energies on meniscus overflow ${ }^{25}$ and transiently constant acceleration of the top pad during capillary self-alignment ${ }^{24}$ are supported by recent experimental evidence.

The model bridges the domain of small displacements of purely elastic models with that of large displacements. The latter is captured by computationally efficient models based on two-dimensional convolutions of the shapes of the bounding pads ${ }^{26}$ (figure 7 ). The analytical formulation of the model, hereby developed for identical square shapes of the pads, can be trivially adapted to rectangular pads. A generalization for polygonal pad shapes of higher order may also be conceived ${ }^{21}$.

Experimental benchmarking of the analytical force model may be envisioned, for instance through the adaptation of an earlier setup for lateral capillary force measurement ${ }^{12}$ which could notably impose a strictly horizontal displacement of the top pad.
Pivotal to the formulation of the model is the energetic description of contact line unpinning and subsequent sliding. Under model assumptions, this has the simple form derived from the Young-Dupré' equation. A similar formulation could be adopted to describe more realistic scenarios involving finite contact angle hysteresis, provided substitutive descriptions of the energy and force balance at the triple contact lines ${ }^{34}$.

\section{ACKNOWLEDGMENTS}

This research has been funded by the Interuniversity Attraction Poles Programme (IAP 7/38 MicroMAST) initiated by the Belgian Science Policy Office.

\section{APPENDIX: MODEL DERIVATION}

In this section we present the full derivation of the analytical model presented in the main text.

With reference to the geometry sketched in figure 1 , we subsume the partial wetting of the surfaces of the pads in considering $0<\theta_{t}<\theta_{b}<\pi / 2$, yielding $u_{1}=h \cot \theta_{b}$ and $u_{2}=h \cot \theta_{t}$ according to eq. 3 . The alternative case of $0<\theta_{b}<\theta_{t}<\pi / 2$ differs only in the sequence of contact line unpinnings over the pads, its formulation being the same upon mutual replacement of $t$ with $b$. Figure 8 provides the reference geometries for the estimation of the updated values $h^{\prime}$ and $h^{\prime \prime}$ of $h(u)$ upon transitions between regimes under conservation of meniscus volume.

The following holds under model assumptions:

1. the surface energy $E(u)$ coincides with the total free energy of the capillary system, and it is invariant under swapping of the surface energies of pads;

2. partial wettability of the surfaces of the pads determines the existence of finite relative displacements $u_{i}$ causing the sequential unpinning of the extremal contact lines (i.e. those whose vertical projection lies outside the opposite pads' surface).

The analytical formulation of the model proceeds from the calculation of the energy $E_{j}(u)$ of the system ${ }^{26}$ for each regime $R_{j}$ determined by sequential unpinning discontinuities. The lateral capillary force $F_{j}(u)$ and stiffness $k_{j}(u)$ of the meniscus are computed by subsequent partial derivatives over $u$ of the energy function.

The energy of the global equilibrium state $R_{0}$ is (up to an additional arbitrary constant):

$$
E_{0}=E\left(u_{0}=0\right)=\underbrace{L^{2}\left(\gamma_{\mathrm{sl}}^{t}+\gamma_{\mathrm{sl}}^{b}\right)}_{\text {pads }}+\underbrace{4 \frac{V}{L} \gamma}_{\text {meniscus }}
$$




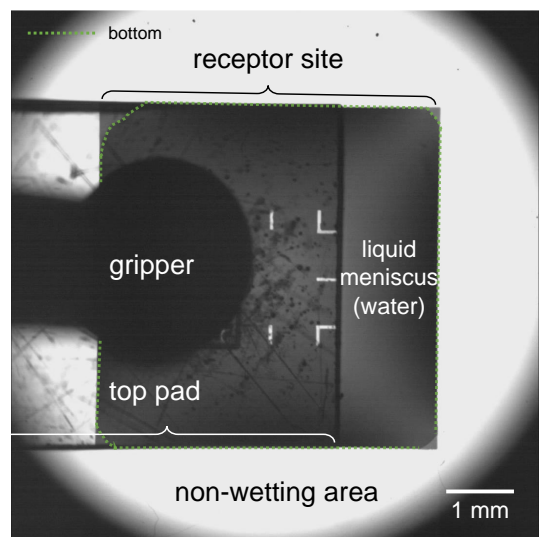

(a) Pre-alignment of top pad by vacuum gripping (no liquid bridge)

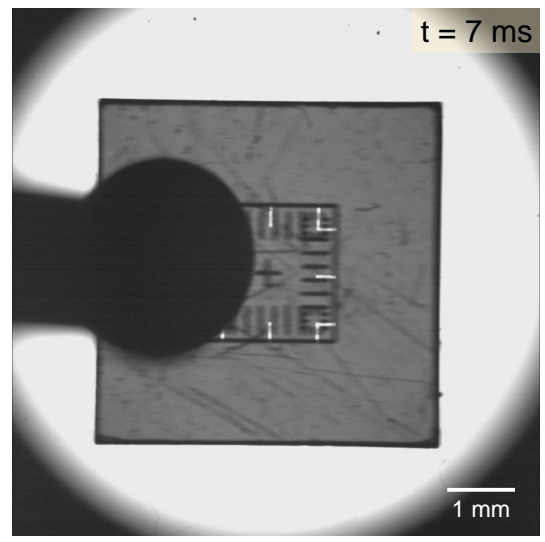

(d) first crossing of receptor site $-R_{0}$

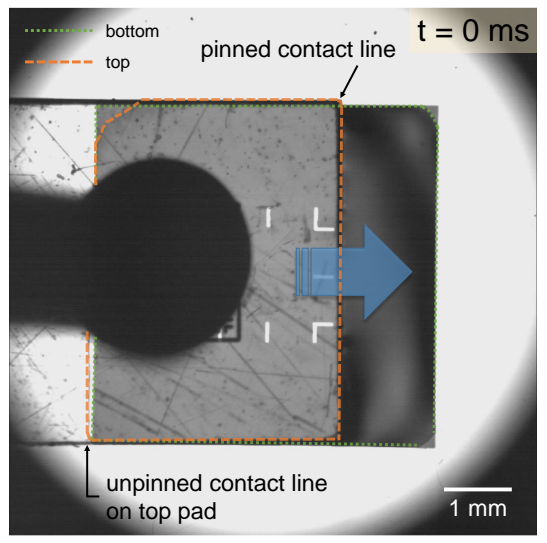

(b) Establishment of liquid bridge and transient wetting - mixed regime

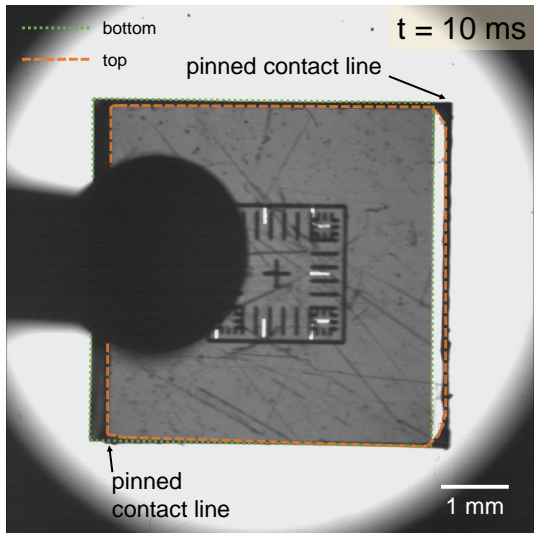

(e) overshoot - fully elastic regime

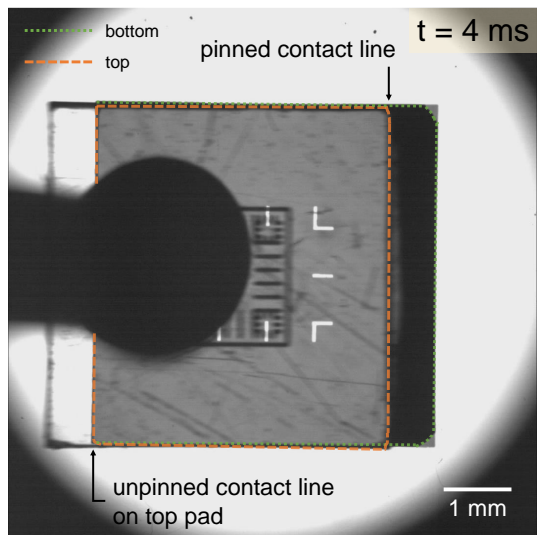

(c) constant acceleration - mixed regime

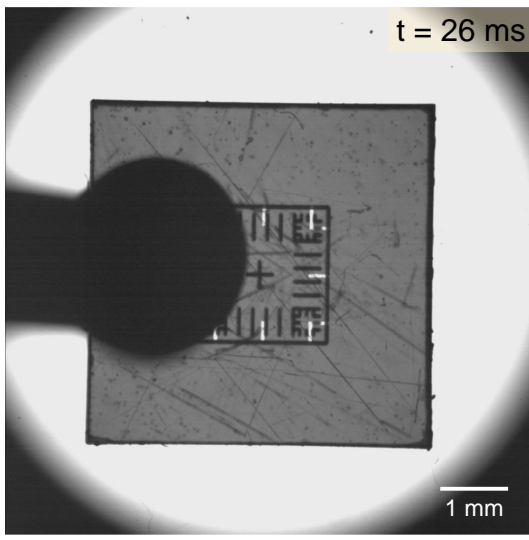

(f) final equilibrium position $-R_{0}$

FIG. 6. Sequential snapshots from high-speed recording of a capillary self-alignment experiment. The transparent top square pad was released from a vacuum gripper onto a chemically patterned and shape-matching receptor site conformally pre-coated with water $\left(L=5 \mathrm{~mm}, h=125 \mu \mathrm{m}(\mathrm{AR}=1 / 40), \theta_{t}=65^{\circ}\right.$ and $\left.\theta_{b}=10^{\circ}\right)$. Top and bottom contact lines are highlighted. Full video available in Supporting Information.

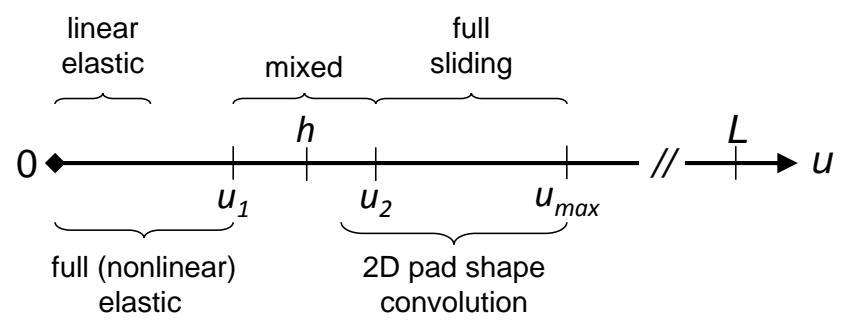

FIG. 7. Qualitative range of validity of capillary force models.

For the deformed states $R_{1}$ and $R_{2}$ :

$$
\begin{aligned}
E_{1} & =E\left(0 \leq u \leq u_{1}\right) \\
& =\underbrace{L^{2}\left(\gamma_{\mathrm{sl}}^{t}+\gamma_{\mathrm{sl}}^{b}\right)}_{\text {pads }}+\underbrace{2 \frac{V}{L} \gamma}_{\text {lateral }}+\underbrace{2 \gamma L \sqrt{h^{2}+u^{2}}}_{\text {front \& rear }} \\
& =E_{0}-2 \frac{V}{L} \gamma+2 \gamma L \sqrt{h^{2}+u^{2}}
\end{aligned}
$$

$$
\begin{aligned}
E_{2} & =E\left(u_{1} \leq u \leq u_{2}\right) \\
& =\underbrace{L^{2} \gamma_{\mathrm{sl}}^{t}}_{\text {top pad }}+\underbrace{L\left(u-u_{1}\right) \gamma_{\mathrm{sv}}^{b}+L\left[L-\left(u-u_{1}\right)\right] \gamma_{\mathrm{sl}}^{b}}_{\text {bottom pad }} \\
& +\underbrace{2 \gamma \frac{V}{L}}_{\text {lateral }}+\underbrace{\gamma L \sqrt{h^{\prime 2}+u_{1}^{2}}}_{\text {rear }}+\underbrace{\gamma L \sqrt{h^{\prime 2}+u^{2}}}_{\text {front }} \\
& =E_{1}\left(u_{1}\right)+\gamma L\left(\sqrt{h^{\prime 2}+u^{2}}-\sqrt{h^{\prime 2}+u_{1}^{2}}\right) \\
& +\left(\gamma_{\mathrm{sv}}^{b}-\gamma_{\mathrm{sl}}^{b}\right) L\left(u-u_{1}\right) \\
& \cong \text { const }+\gamma L \sqrt{h^{2}+u^{2}}+\gamma L\left(u-u_{1}\right) \cos \left(\theta_{b}\right)
\end{aligned}
$$

using eq. 1 and the following approximation for the constancy of $h$ (see Figure 8(a)):

$$
\begin{aligned}
h^{\prime} & =h \frac{L}{L-\frac{u-u_{1}}{2}} \\
& =h\left(1+\frac{u-u_{1}}{2 L}\right)+o^{2}\left(u-u_{1}\right) \\
& \cong h \text { for } u \ll 2 L+u_{1}=2 L+h \cot \theta_{b}
\end{aligned}
$$




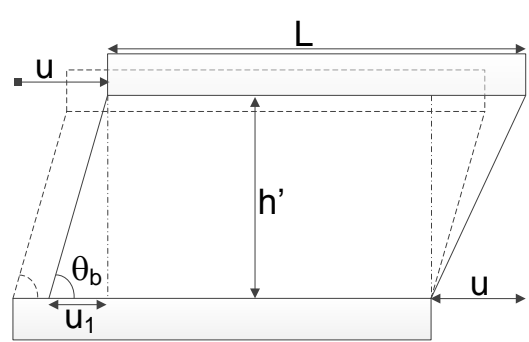

(a) Sketch for $h^{\prime}$

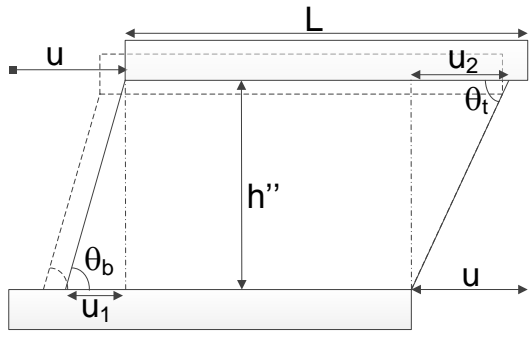

(b) Sketch for $h^{\prime \prime}$

FIG. 8. Sketches (not to scale) for geometrical estimation of $h^{\prime}$ and $h^{\prime \prime}$ under model assumptions upon transitions between adjacent regimes: (a) from $R_{1}$ to $R_{2}$, (a) from $R_{2}$ to $R_{3}$.

Similarly for $R_{3}$ :

$$
\begin{aligned}
E_{3} & =E\left(u_{2} \leq u<u_{\text {max }}\right) \\
& =\underbrace{2 \frac{V}{L} \gamma}_{\text {lateral }}+\underbrace{\gamma L \sqrt{h^{\prime 2}+u_{1}^{2}}}_{\text {rear }}+\underbrace{\gamma L \sqrt{h^{\prime 2}+u_{2}^{2}}}_{\text {front }} \\
& +\underbrace{L\left(u-u_{1}\right) \gamma_{\mathrm{sv}}^{b}+L\left[L-\left(u-u_{1}\right)\right] \gamma_{\mathrm{s}}^{b}}_{\text {bottom pad }} \\
& +\underbrace{L\left(u-u_{2}\right) \gamma_{\mathrm{sv}}^{t}+L\left[L-\left(u-u_{2}\right)\right] \gamma_{\mathrm{sl}}^{t}}_{\text {top pad }} \\
& \cong E_{2}\left(u_{2}\right)+\gamma L\left(u-u_{2}\right)\left(\cos \theta_{b}+\cos \theta_{t}\right)
\end{aligned}
$$

using the approximation (see Figure 8(b)):

$$
\begin{aligned}
h^{\prime \prime} & =h \frac{L}{L-\left(u-\frac{u_{1}+u_{2}}{2}\right)} \\
& \cong h \text { for } u \ll L+\frac{u_{1}+u_{2}}{2}=L+\frac{h}{2}\left(\cot \theta_{b}+\cot \theta_{t}\right)
\end{aligned}
$$

Eq. 11, more stringent than eq. 9, sets the strict limit of validity of the model over $u$ under the assumptions of constant $V$ and $h$. This condition assumes and is consistent with choices of coupled pairs of $\theta_{*}$ satisfying the condition set by eq. 5 for overflow-less transition between adjacent capillary regimes. Eq. 11 defines $u_{\max }\left(\theta_{*}, h\right)$ and relates it to the pad size $L$ rather than to $h(L \gg h$ in general) as in purely elastic models. Given $L=1 \mathrm{~mm}$, $h$ and $\theta_{*}$, the relative errors in capillary force estimates between analytical and numerical models for each of the cases reported in Table I of the main text were evaluated for the corresponding value:

$$
u_{\max }=\frac{1}{10}\left[L+\frac{h}{2}\left(\cot \theta_{b}+\cot \theta_{t}\right)\right]
$$

From eqs. 7,8 and 10 it follows respectively:

$$
\begin{aligned}
& R_{1}\left\{\begin{aligned}
F_{1}(u) & =-\frac{\partial E_{1}(u)}{\partial u}=-2 \gamma L \frac{u}{\sqrt{h+2+u^{2}}} \\
k_{1}(u) & =-\frac{\partial F_{1}(u)}{\partial u}=\frac{\partial^{2} E_{1}(u)}{\partial u^{2}} \\
& =2 \gamma L\left(\frac{1}{\sqrt{h^{2}+u^{2}}}-\frac{u^{2}}{\left(h^{2}+u^{2}\right)^{\frac{3}{2}}}\right)
\end{aligned}\right. \\
& R_{2}\left\{\begin{aligned}
F_{2}(u) & =-\frac{\partial E_{2}(u)}{\partial u}=-\gamma L\left(\frac{u}{\sqrt{h+2+u^{2}}}+\cos \theta_{b}\right) \\
k_{2}(u) & =-\frac{\partial F_{2}(u)}{\partial u}=\frac{\partial^{2} E_{2}(u)}{\partial u^{2}} \\
& =\gamma L\left(\frac{1}{\sqrt{h^{2}+u^{2}}}-\frac{u^{2}}{\left(h^{2}+u^{2}\right)^{\frac{3}{2}}}\right) \\
& =\frac{k_{1}(u)}{2}
\end{aligned}\right. \\
& R_{3}\left\{\begin{aligned}
F_{3}(u) & =-\frac{\partial E_{2}(u)}{\partial u}=-\gamma L\left(\cos \theta_{b}+\cos \theta_{t}\right) \\
k_{3}(u) & =-\frac{\partial F_{3}(u)}{\partial u}=\frac{\partial^{2} E_{3}(u)}{\partial u^{2}} \\
& =0
\end{aligned}\right.
\end{aligned}
$$

The formulation is consistent with energy and force continuity across adjacent domains, since $E_{i+1}\left(u_{i}\right)=$ $E_{i}\left(u_{i}\right)$ and $F_{i+1}\left(u_{i}\right)=F_{i}\left(u_{i}\right)$ hold for all $i=0,1,2$. Note that for $\left.R_{1}\right|_{0<u<h}$ the small displacement values $F_{1}=-2 \gamma L \frac{u}{h}$ and $k_{1}=2 \gamma \frac{L}{h}$ of linear elastic models are recovered ${ }^{9,12,20,29}$. Conversely, the absence of elastic work in $R_{3}$ is evidenced by the null constant value of $k_{3}$. Also, $\theta_{t} \rightarrow \theta_{b}$ implies a singular domain for $R_{2}$ as $u_{2} \rightarrow u_{1}$. Particularly, for the limiting case of full wetting of both pads-i.e. for $\theta_{b}=\theta_{t}=0-u_{1} \rightarrow \infty$, i.e. the domain of $R_{1}$ extends indefinitely. The purely elastic regime is thus recovered, whereby partial dewetting of the surface of the pads is not possible. In this ideal condition further model convergence is given by $\left.F_{1}\right|_{u \gg h}=\left.F_{3}\right|_{\theta_{b}=\theta_{t}=0}$. Plots of energy, gap and restoring force versus $u$ for $\mathrm{AR}=1 / 20$ and $1 / 10(h=50$ and $100 \mu \mathrm{m}$, respectively) are shown in figures 9 through 14 complementing those for $A R=1 / 40$ presented in the main text.

Finally, by considering meniscus confinement within the pads by chemical contrast (figure 2(b)) rather than by topographical step, the formal derivation shown above can be adapted to account for the case of liquid bridge overflow $^{26}$. Overflow is here supposed to take place beyond the edge of the bottom pad onto an adjacent and less wettable surface (see figure 15). The case of overflow beyond the top pad is energetically equivalent. We assume that the two (pad and adjacent) surfaces are at 


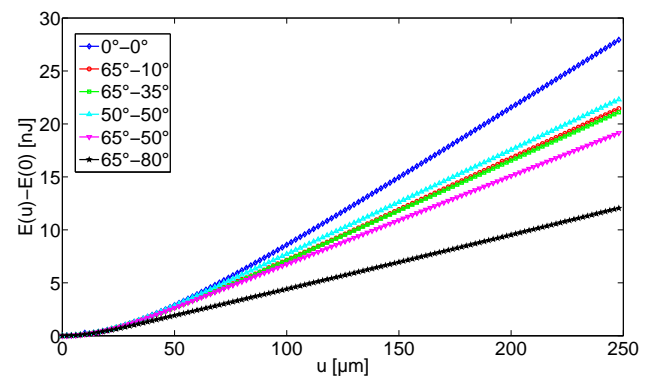

(a) total energy $E(u)-E(0)$ versus $u$

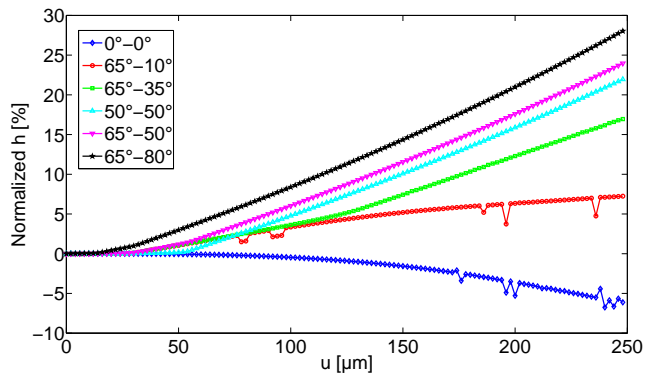

(b) normalized gap $\left(h-h_{0}\right) / h_{0}$ versus $u$

FIG. 9. SE simulations for $h_{0}=50 \mu \mathrm{m}(\mathrm{AR}=1 / 20)$ parameterized by $\theta_{b}-\theta_{t}$ pairs.

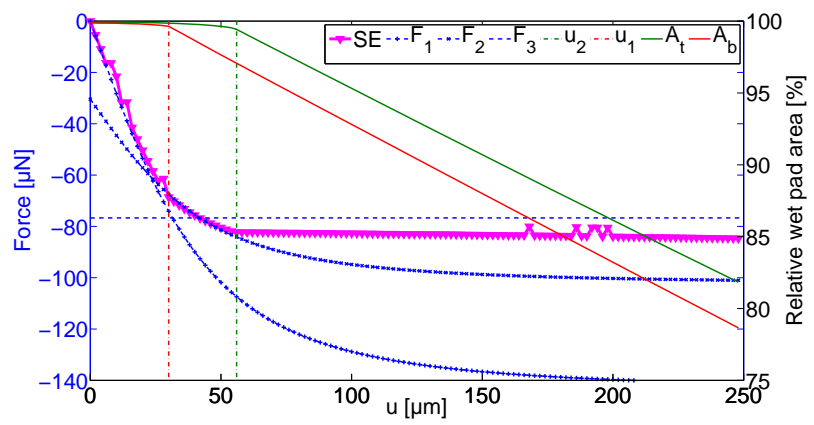

FIG. 10. SE simulation and analytical fit of $F(u)$ versus $u$ for $h_{0}=50 \mu \mathrm{m}(\mathrm{AR}=1 / 20)$ with $\theta_{b}=65^{\circ}$ and $\theta_{t}=50^{\circ}$. The relative error for $F\left(u=u_{\max }\right)$ is $7.4 \%$.

the same level, and that the energetic barrier to overflow is only chemical in nature. For the less wettable surface the validity of a specific Young-Dupré equation is also assumed, yielding a contact angle $\theta_{b}^{o f}>\pi / 2>\theta_{b}$. The overflow happens when the edge angle of the meniscus reaches the advancing value of the contact angle on the adjacent surface (hereby again assumed to coincide with its static value $\theta_{b}^{o f}$ ), prompting the unpinning of the contact line toward the adjacent surface. Unpinning takes place for $u \geq u_{o f}$ and signals the transition to a regime akin to either the mixed $\left(R_{2}^{o f}\right)$ or the full sliding one $\left(R_{3}^{o f}\right) . u_{o f}$ can be either larger or smaller than $u_{1}$, yet not larger than $u_{2}$ because in $R_{3}$ the inclinations of both perpendicular sides of the meniscus remain constant. For the former case of $0<u_{o f}<u_{1}$ (fig. 15(a)):

$$
R_{2}^{o f}\left\{\begin{aligned}
F_{2}^{o f}(u) & =-\frac{\partial E_{2}^{o f}(u)}{\partial u}=-\gamma L\left(\frac{u}{\sqrt{h+2+u^{2}}}-\cos \theta_{b}^{o f}\right) \\
k_{2}^{o f}(u) & =-\frac{\partial F_{2}^{o f}(u)}{\partial u}=\frac{\partial^{2} E_{2}^{o f}(u)}{\partial u^{2}} \\
& =\gamma L\left(\frac{1}{\sqrt{h^{2}+u^{2}}}-\frac{u^{2}}{\left(h^{2}+u^{2}\right)^{\frac{3}{2}}}\right) \\
& =\frac{k_{1}(u)}{2}
\end{aligned}\right.
$$

For the latter case of $u_{1}<u_{o f}$ (fig. 15(b)):

$$
R_{3}^{o f}\left\{\begin{aligned}
F_{3}^{o f}(u) & =-\frac{\partial E_{2}^{o f}(u)}{\partial u}=-\gamma L\left(\cos \theta_{b}-\cos \theta_{b}^{o f}\right) \\
k_{3}^{o f}(u) & =-\frac{\partial F_{3}^{o f}(u)}{\partial u}=\frac{\partial^{2} E_{3}^{o f}(u)}{\partial u^{2}} \\
& =0
\end{aligned}\right.
$$

${ }^{1}$ D. J. Broesch and J. Frechette, "From Concave to Convex: Capillary Bridges in Slit Pore Geometry," Langmuir 28, 15548 - 15554 (2012).

${ }^{2}$ D. J. Broesch, F. Dutka, and J. Frechette, "Curvature of capillary bridges as a competition between wetting and confinement," Langmuir 29, 15558-15564 (2013).

${ }^{3}$ P. Lambert, ed., Surface tension in microsystems (Springer, 2013).

${ }^{4} \mathrm{P}$. Lambert, Capillary forces in microassembly (Springer, 2007). ${ }^{5}$ H. O. Jacobs, A. R. Tao, A. Schwartz, D. H. Gracias, and G. M. Whitesides, "Fabrication of a cylindrical display by patterned assembly," Science 296, 323-325 (2002).

${ }^{6}$ R. J. Knuesel and H. O. Jacobs, "Self-assembly of microscopic chiplets at a liquid-liquid-solid interface forming a flexible segmented monocrystalline solar cell," Proc. Natl. Acad. Sci. 107, 993-998 (2010).

${ }^{7}$ T. Fukushima, E. Iwata, Y. Ohara, M. Murugesan, J. Bea, K. Lee, T. Tanaka, and M. Koyanagi, "Multichip-to-wafer threedimensional integration technology using chip self-assembly with excimer lamp irradiation," IEEE Trans. Electron. Dev. 59, 29562963 (2012).

${ }^{8}$ M. Mastrangeli, W. Ruythooren, J.-P. Celis, and C. van Hoof, "Challenges for capillary self-assembly of microsystems," IEEE Trans. on Comp. Pack. Man. Tech. 1, 133-149 (2011).

${ }^{9}$ J. Berthier, K. Brakke, F. Grossi, L. Sanchez, and L. Di Cioccio, "Self-alignment of silicon chips on wafers: A capillary approach," J. Appl. Phys. 108, 054905 (2010).

${ }^{10}$ K. L. Scott, R. T. Howe, and C. J. Radke, "Model for micropart planarization in capillary-based microassembly," in 12th Int. Conf. on Solid-State Sensors, Actuators and Microsystems (Transducers), Vol. 2 (2003) pp. 1319-1322.

${ }^{11}$ S. Abbasi, A. Zhou, R. Baskaran, and K. F. Böhringer, "Part tilting in capillary-based self-assembly: Modeling and correction methods," in IEEE 21st Int. Conf. on Micro Electro Mechanical Systems (MEMS 2008) (2008) pp. 1060-1063.

${ }^{12}$ M. Mastrangeli, J.-B. Valsamis, C. van Hoof, J.-P. Celis, and P. Lambert, "Lateral capillary forces of cylindrical fluid menisci: a comprehensive quasi-static study," J. Micromech. Microeng. 20, 075041 (2010).

${ }^{13}$ A. Takei, K. Matsumoto, and I. Shimoyama, "Capillary torque caused by a liquid droplet sandwiched between two plates," Langmuir 26, 2497-2504 (2010).

${ }^{14}$ D. J. Broesch, E. Shiang, and J. Frechette, "Role of substrate aspect ratio on the robustness of capillary alignment," Appl. Phys. Lett. 104, 081605 (2014).

${ }^{15}$ S. K. Patra and Y. C. Lee, "Quasi-Static Modeling of the SelfAlignment Mechanism in Flip-Chip SolderingPart I: Single Solder Joint," J. Electron. Packag. 113, 337-342 (1991).

${ }^{16}$ C. Lenders, M. Gauthier, R. Cojan, and P. Lambert, "ThreeDOF Microrobotic Platform Based on Capillary Actuation," IEEE Trans. Robot. 28, $1157-1161$ (2012). 


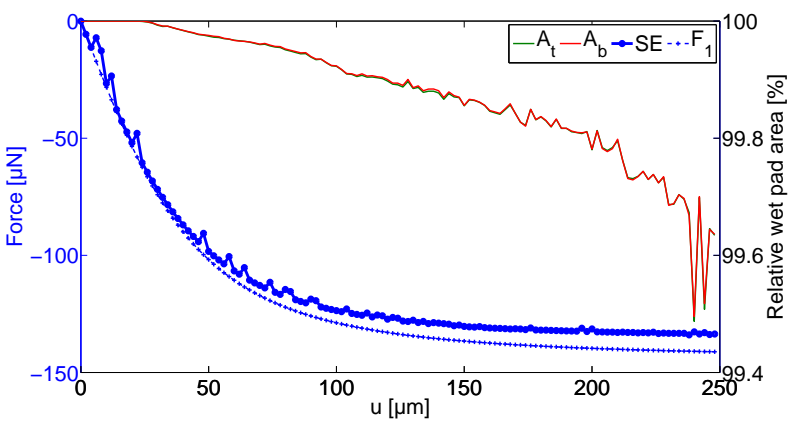

(a) $\theta_{b}=\theta_{t}=0^{\circ}$

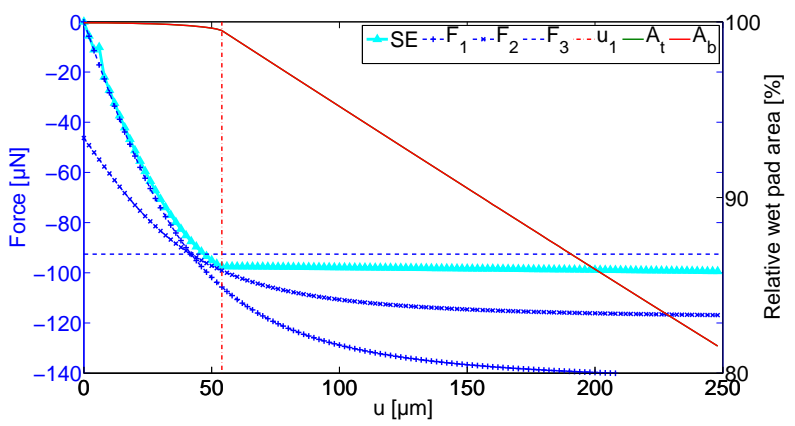

(c) $\theta_{b}=\theta_{t}=50^{\circ}$

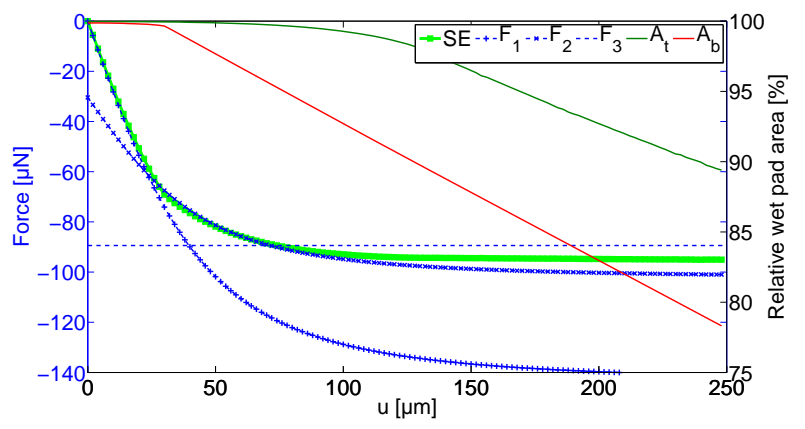

(b) $\theta_{b}=65^{\circ}, \theta_{t}=35^{\circ}$

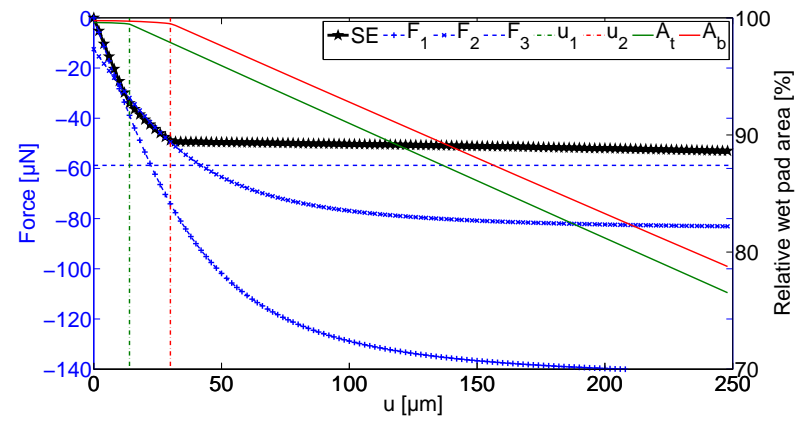

(d) $\theta_{b}=65^{\circ}, \theta_{t}=80^{\circ}$

FIG. 11. Capillary forces predicted for $h=50 \mu \mathrm{m}(\mathrm{AR}=1 / 20)$ and various combinations of $\theta_{*}$. (a) Perfect wetting (no contact line unpinning), (b) the three sequential regimes, first unpinning on bottom pad, (c) absence of second regime for $\theta_{b}=\theta_{t}$ $\left(u_{1}=u_{2}=u_{d}, F_{1}\left(u_{d}\right)=F_{2}\left(u_{d}\right)=F_{3}\left(u_{d}\right)\right)$, (d) the three sequential regimes, first unpinning on top pad.

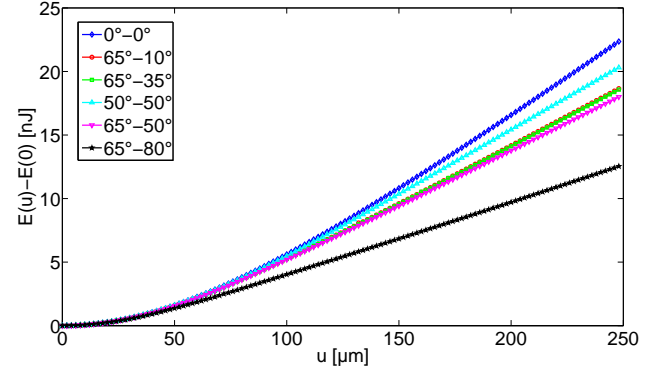

(a) total energy $E(u)-E(0)$ versus $u$

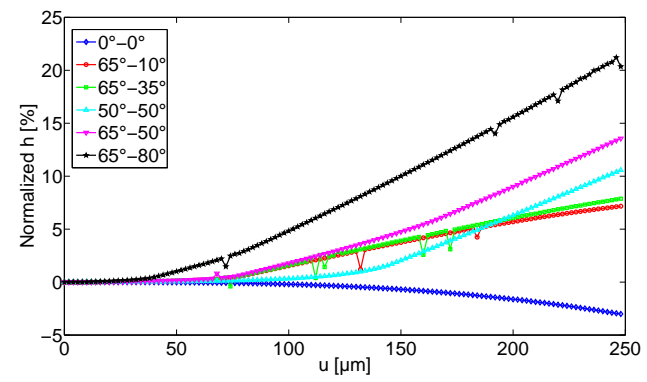

(b) normalized gap $\left(h-h_{0}\right) / h_{0}$ versus $u$

FIG. 12. SE simulations for $h_{0}=50 \mu \mathrm{m}(\mathrm{AR}=1 / 20)$ parameterized by $\theta_{b}-\theta_{t}$ pairs.

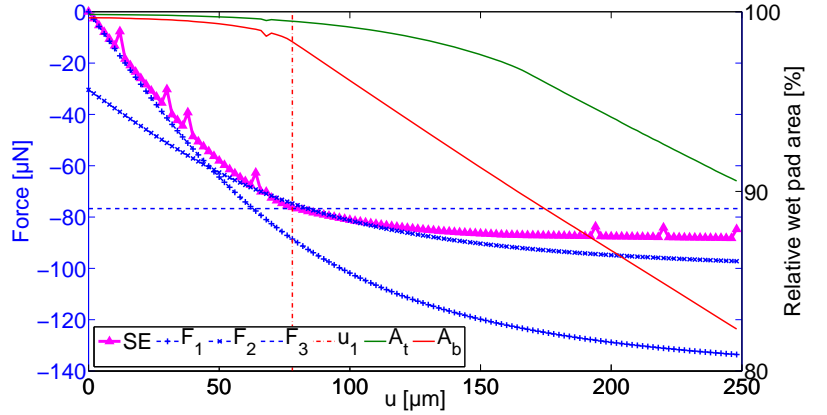

FIG. 13. SE simulation and analytical fit of $F(u)$ versus $u$ for $h_{0}=100 \mu \mathrm{m}(\mathrm{AR}=1 / 10)$ with $\theta_{b}=65^{\circ}$ and $\theta_{t}=50^{\circ}$. Relative error for $F\left(u=u_{\max }\right)$ is $7.4 \%$.

${ }^{17}$ J.-B. Valsamis, M. Mastrangeli, and P. Lambert, "Vertical excitation of axisymmetric liquid bridges," Eur. J. Mech. B-Fluid 38, 47-57 (2013).

${ }^{18}$ K. Sato, K. Itoa, S. Hata, and A. Shimokohbe, "Self-alignment of microparts using liquid surface tension-behavior of micropart and alignment characteristics," Precis. Eng. 27, 42-50 (2003).

${ }^{19}$ V. Sariola, M. Jääskeläinen, and Q. Zhou, "Hybrid microassembly combining robotics and water droplet self-alignment," IEEE Trans. Robot. 26, $965-977$ (2010).

${ }^{20}$ P. Lambert, M. Mastrangeli, J.-B. Valsamis, and G. Degrez, "Spectral analysis and experimental study of lateral capillary dynamics for flip-chip applications," Microfluid. Nanofluid. 9, 797807 (2010). 


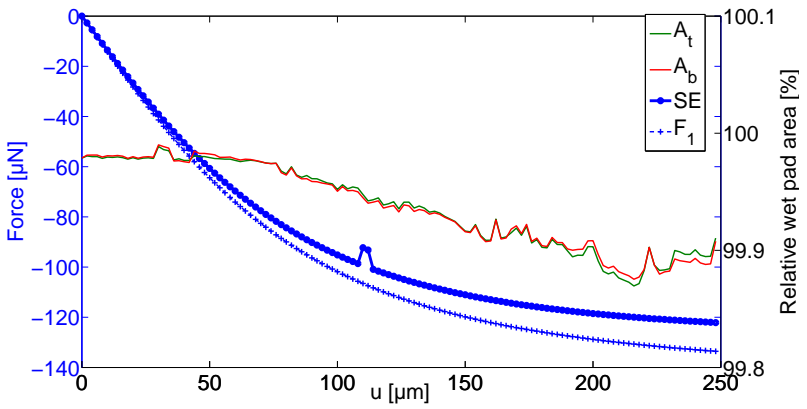

(a) $\theta_{b}=\theta_{t}=0^{\circ}$

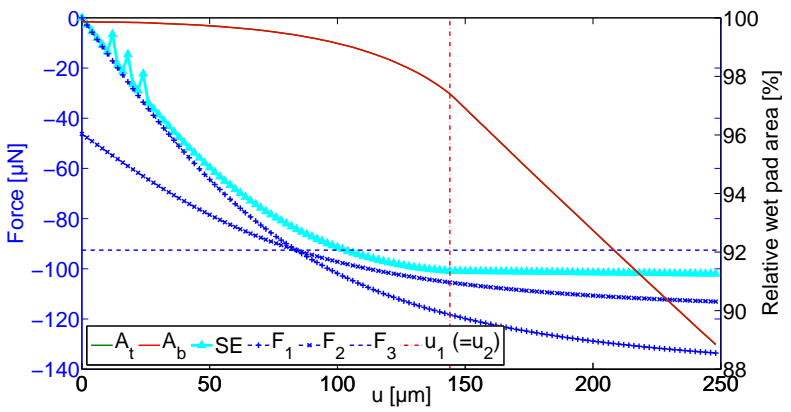

(c) $\theta_{b}=\theta_{t}=50^{\circ}$

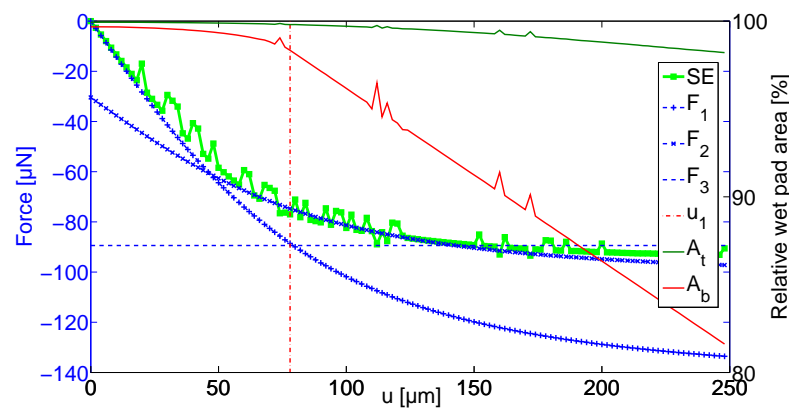

(b) $\theta_{b}=65^{\circ}, \theta_{t}=35^{\circ}$

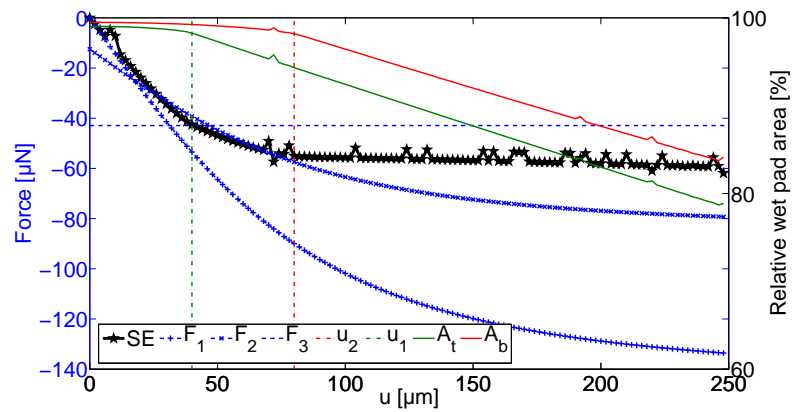

(d) $\theta_{b}=65^{\circ}, \theta_{t}=80^{\circ}$

FIG. 14. Capillary forces predicted for $h=100 \mu \mathrm{m}(\mathrm{AR}=1 / 10)$ and various combinations of $\theta_{b}$ and $\theta_{t}$ values. (a) Perfect wetting (no contact line unpinning), (b) the three sequential regimes, first unpinning on bottom pad, (c) absence of second regime for $\theta_{b}=\theta_{t}\left(u_{1}=u_{2}=u_{d}, F_{1}\left(u_{d}\right)=F_{2}\left(u_{d}\right)=F_{3}\left(u_{d}\right)\right)$, (d) the three sequential regimes, first unpinning on top pad.

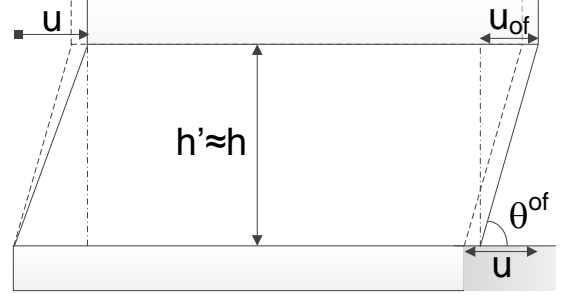

(a) Sketch for $0<u_{o} f<u_{1}$

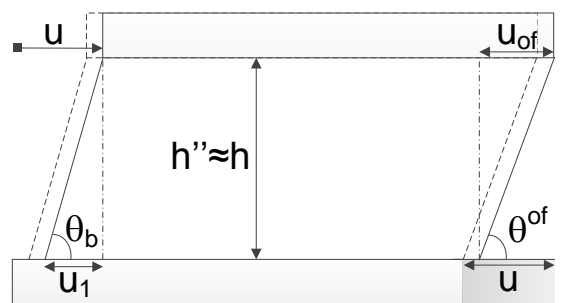

(b) Sketch for $u_{1}<u_{o} f$

FIG. 15. Transitions to overflow regimes: (a) from $R_{1}$ to $R_{2}^{o f}$, (a) from $R_{2}$ to $R_{3}^{o f}$.
${ }^{21}$ J. Berthier, S. Mermoz, K. Brakke, L. Sanchez, C. Frétigny, and L. Di Cioccio, "Capillary self-alignment of polygonal chips: a generalization for the shift-restoring force," Microfluid. Nanofluid. 14, 845-858 (2013).

${ }^{22} \mathrm{~S}$. Gao and Y. Zhou, "Self-alignment of micro-parts using capillary interaction: Unified modeling and misalignment analysis," Microelectron. Reliab. 53, 1137-1148 (2013).

${ }^{23}$ G. Arutinov, E. C. P. Smits, M. Mastrangeli, G. Van Heck, J. van den Brand, H. F. M. Schoo, and A. Dietzel, "Capillary self-alignment of mesoscopic foil components for sensor-systemsin-foil," J. Micromech. Microeng. 22, 115022 (2012).

${ }^{24}$ G. Arutinov, M. Mastrangeli, E. C. P. Smits, H. F. M. Schoo, J. Brugger, and A. Dietzel, "Dynamics of capillary self-alignment for mesoscopic foil devices," Appl. Phys. Lett. 102, 144101 (2013).

${ }^{25}$ G. Arutinov, M. Mastrangeli, E. C. P. Smits, G. Van Heck, J. M. J. den Toonder, and A. Dietzel, "Foil-to-foil system integration through capillary self-alignment directed by laser patterning," IEEE J. Microelectromech. Sys. to appear (2014).

${ }^{26}$ J. Lienemann, A. Greiner, J. G. Korvink, X. Xiong, Y. Hanein, and K. F. Böhringer, "Sensor update 13," (Wiley-VCH, 2004) Chap. Modeling, Simulation, and Experimentation of a Promising New Packaging Technology: Parallel Fluidic Self-Assembly of Microdevices, pp. 3-43.

${ }^{27}$ J. Lienemann, D. Weiss, A. Greiner, D. Kauzlaric, O. Grünert, and J. G. Korvink, "Insight into the micro scale dynamics of a micro fluidic wetting-based conveying system by particle based simulation," Microsyst. Technol. 18, 523-530 (2012).

${ }^{28} \mathrm{~J}$. Berthier and K. Brakke, The physics of microdroplets (John Wiley \& Sons and Scrivener Publishing, 2012).

${ }^{29}$ C. G. Tsai, C. M. Hsieh, and J. A. Yeh, "Self-alignment of microchips using surface tension and solid edge," Sensor. Actuat. A-Phys. 139, 343-349 (2007). 
${ }^{30}$ V. Limatainen, V. Sariola, and Q. Zhou, "Controlling liquid spreading using microfabricated undercut edges," Adv. Mater. 25, 2275-2278 (2013).

${ }^{31}$ K. Brakke, "The surface evolver," Exp. Math. 1, 141-165 (1992).

${ }^{32} \mathrm{C}$. Lanczos, The variational principles of mechanics (Dover, 1970).

${ }^{33}$ J. Berthier, K. Brakke, L. Sanchez, and L. di Cioccio, "Self- alignment of silicon chips on wafers: a numerical investigation of the effect of spreading and wetting," Sensors \& Transducers Journal 13, 44-52 (2011)

${ }^{34}$ J. H. Snoeijer and B. Andreotti, "Moving contact lines: Scales, regimes and dynamical transitions," Annu. Rev. Fluid Mech. 45, 269-292 (2013). 